\title{
L'épopée de la dialyse et de la transplantation
}

\section{Jean Martin}

Membre de la rédaction

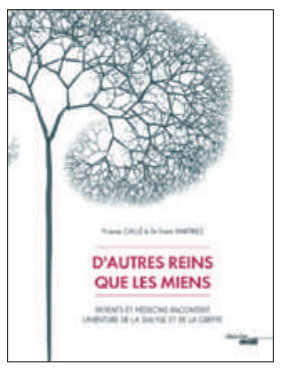

Yvanie Caillé,

Frank Martinez

D'autres reins

que les miens

Patients et médecins racontent l'aventure de la dialyse et de la greffe. Paris: Le cherche midi; 2014 .

223 pages. $33.70 \mathrm{CHF}$. ISBN 978-2-7491-3522-9
Cet ouvrage brosse un portrait multidimensionnel de la prise en charge de l'insuffisance rénale au cours des soixante dernières années en France (avec des notations internationales). Seize témoignages ont été recueillis par les auteurs, une ingénieure malade rénale, aujourd'hui transplantée, et un médecin de l'Hôpital Necker, à Paris. Avec une utile préface d'un sociologue qui a donné un de ses reins.

\section{Une histoire médicale et humaine mais aussi politique et organisationnelle}

Cette saga va de 1950, alors que rien d'efficace ne peut être fait pour les patients urémiques qui décèdent en quelques jours ou semaines, à aujourd'hui, où des millions de personnes dans le monde suivent une dialyse chronique et où des centaines de milliers ont été greffées. Ces chiffres ne doivent pas faire oublier les difficultés, au cours de décennies, de celles et ceux qui ont vécu des trajectoires faites de maladie, de souffrance, d'espoirs et de déceptions. Et qui, avec les médecins et autres scientifiques, les psychologues, les assistantes sociales, plus tard les coordinateurs de transplantation, sont les acteurs (les héros pourrait-on dire) de cette histoire.

Beaucoup de notations du registre historique sur les développements en France. Rappel que, inspirée par les travaux de Belding Scribner, de Seattle, la dialyse chronique a été développée à Lyon, par le professeur Jules Traeger, avec quelque cinq ans d'avance sur les services parisiens. Paris où le patron "tout-puissant» (sic) était Jean Hamburger, visionnaire, proche des politiques et des médias, qui à l'époque concentrait ses recherches sur la possibilité de la greffe plutôt que sur la dialyse. Le premier congrès mondial de néphrologie s'est tenu en 1960 à Evian.

Rappel d'un véritable fléau, avant que la France ne connaisse la loi Veil de 1975 dépénalisant l'interruption de grossesse: «A l'Hôtel-Dieu, vers 1960, la dialyse était réservée à ceux dont les reins étaient bloqués temporairement et qui pourraient reprendre une vie normale à l'issue de quelques séances de dialyse. Ces insuffisances aiguës étaient provoquées principalement par des avortements clandestins ayant entraîné une infection généralisée. En moins de trois ans, nous avons traité plus de 500 victimes d'infections dues à un tel avortement. Presque toutes ont survécu. Avant, la plupart auraient succombé» (le néphrologue Claude Jacobs).

Sur les résistances vues dans les débuts: «Les progrès sont nés de transgressions successives. Prendre un organe sur un mort ou sur un vivant pour le greffer, filtrer le sang dans une machine de manière itérative, étaient des actes difficilement concevables à l'époque. Ce qui semble aujourd'hui banal apparaissait comme autant de violations choquantes des frontières du corps humain ou de recours abusifs à la technique médicale. Fallait-il franchir ces limites?»

\section{Evolution des relations entre médecins, institutions et malades}

Problématique présente dans de nombreux témoignages. Ainsi à propos du paternalisme médical de l'époque, parfois autoritaire et qui se voyait omniscient. Progressivement, montée d'une volonté de voix au chapitre et de codécision chez les malades (avec des accents qui font penser à ce qu'on a vécu depuis les années 1980 pour le VIH/sida).

Régis Volle, 71 ans aujourd'hui, a vécu tout un périple médical personnel et a fondé la Fédération nationale d'aide aux insuffisants rénaux (Fnair), première association de patients concernés: «Très tôt, la Fnair a revendiqué le droit pour le malade d'être traité, d'accéder à la survie, mais aussi de choisir son traitement à partir d'éléments factuels, médicaux, pratiques, ainsi qu'en fonction de ses aspirations personnelles.» Question de la transparence dans le dialogue: «Au fil $\mathrm{du}$ temps, l'information est probablement devenue plus honnête, lorsque les résultats se sont améliorés. Mais, jusqu'au début des années 1980, il y a eu une réelle omertà sur le taux d'échecs des greffes et la mortalité des patients.» A noter que, pendant des années, cette fédération s'est montrée réservée vis-à-vis de la greffe, puis les progrès réalisés ont modifié sa position. Volle rappelle comment, en 1978, la ministre de la santé Simone Veil l'a nommé, lui laïc, membre de la Commission nationale d'hémodialyse et de transplantation et qu'il y a été accueilli par ces mots du président (médecin): «Monsieur, nous avons vu cet arrêté [décision] de la ministre, mais nous ne comprenons pas ce que vous faites là.» 
Des attitudes discutables: «Les postes de dialyse ont eu beau se multiplier dans l'Hexagone, l'idée que les patients doivent mériter leur traitement, exprimer de la reconnaissance et obéir aveuglement à toutes les injonctions médicales s'est transmise de génération en génération", dit le préfacier $\mathrm{Ch}$. Baudelot.

Question de «human touch» par rapport aux aspects techniques. "A l'heure où la néphrologie est devenue de plus en plus scientifique, l'œil du néphrologue est parfois davantage attiré par l'écran de l'ordinateur que par le regard du patient.» Les trajectoires de traitement sont toujours lourdes (y compris par la règle des trois séances hebdomadaires de quatre heures), durant des années, en attente d'un organe. Dans le registre de l'empathie, on est marqué par le texte du Dr Janine Bédrossian et celui du Dr Elizabeth Tomkiewicz, fille du pédopsychiatre connu Stanislas T., dont elle reprend le concept d'«attitude authentiquement affective».

A propos d'un malade de 77 ans qui ne veut plus du régime «officiel» de trois séances par semaine, E. Tomkiewicz s'arrange, non sans difficulté, pour qu'il ait deux séances: «Il arrive un âge où la qualité de vie et les choix du patient doivent primer sur d'autres considérations. Mais ce constat n'entre que lentement dans la culture des néphrologues.» Le même médecin: «Un autre patient avait fait le choix de deux séances par semaine. Quand il partait en vacances, le centre susceptible de l'accueillir disait: «Ici c'est trois séances ou rien.) Aucune souplesse, aucune adaptation.» Raisons de cette rigidité? Certainement pas seulement de nature médicale mais aussi pour le confort des soignants et des institutions, pour ne pas bousculer les routines voire éviter des réactions de type syndical.

\section{Promouvoir le don d'organes}

Position du Dr Tenaillon, réanimateur qui a intégré l'Agence française de la biomédecine, qui supervise le domaine: «Lorsque je reviens sur ces décennies, ce qui me semble le plus marquant est que le don d'organes après la mort est passé du statut de pratique exceptionnelle, transgressive, à celui de choix de société [...] Il est légitime de tout faire pour soigner les gens, y compris d'avoir recours aux organes d'une autre personne... Le prélèvement d'organes est désormais défini comme un acte de soins [...] j'espère que la prochaine étape sera celle de l'ancrage en profondeur d'une culture du don.»

A propos des démarches pour favoriser les dons, tâche complexe: «Il s'agit donc d'apprendre aux équipes de coordination à parler à des familles en détresse, à rester en toute circonstance dans une logique d'huma- nité, tout en gardant en tête que l'objectif est de parvenir au don. Il existe des techniques de négociation, sans parler de manipulation, qui peuvent s'appliquer à ces entretiens. L'exemple le plus simple est celui de l'empathie.»

Parole d'une mère qui donné un rein à son fils: «J'ai vite compris que recevoir ce rein n'était pas chose aisée pour Bruno. [Ce long cheminement] était probablement une nécessité pour lui. Ce temps, il en avait besoin pour accepter le don, physiquement et moralement. Il faut être généreux pour recevoir, bien plus que pour donner.»

Sur la question, discutée en Suisse récemment encore, des modalités de consentement au don d'organe (présumé ou au contraire qui doit être explicite): «Le don et la greffe font désormais partie des choix de notre société. S'il est essentiel de respecter les libertés individuelles, il est tout aussi important de rappeler que notre société est basée sur le principe de solidarité. Dans ce contexte, si chacun veut pouvoir bénéficier d'une greffe pour lui-même ou un de ses proches en cas de nécessité, il faut aussi que chacun soit prêt à donner le cas échéant; il s'agit d'un altruisme du type donnant-donnant» (Dr Tenaillon). Le débat sur ce point n'est probablement pas clos!

\section{En résumé}

Cette présentation de la "scène» française de l'insuffisance rénale et de sa prise en charge est pleine d'enseignements - et d'émotions parfois. A l'interface de la recherche, des pratiques de soins, d'une médecine sociale qui cherche à atteindre tous ceux qui en ont besoin, des budgets de la santé et de dimensions humaines majeures. De plus, D'autres reins que les miens fait preuve d'une estimable cohérence du style, par la rédaction des récits par les deux auteurs. Des notes de bas de page expliquent les termes scientifiques et font que, tout en étant de grand intérêt pour les professionnels eux-mêmes, l'ouvrage est aisément compris de tout lecteur intéressé.

Dans la conclusion, ce bémol: «Nos regards sur le passé nous incitent à ne pas nous contenter de ce qui a été acquis mais, au contraire, à continuer à progresser pour tenter de permettre aux patients de vivre mieux. Peu de très grands progrès ont été réalisés, ces vingt dernières années, dans le domaine de la dialyse. Rien qui ait modifié ses contraintes ou amélioré sensiblement la qualité ou l'espérance de vie des malades. Pourtant les soulager de ce fardeau, les libérer de cette (prison sans barreaux), tout en faisant diminuer les coûts associés, aurait dû apparaître comme un impératif.» 